Vol.59: e16161055, January-December 2016 http://dx.doi.org/10.1590/1678-4324-2016161055 ISSN 1678-4324 Online Edition
BRAZILIAN ARCHIVES OF BIOLOGY AND TECHNOLOGY

AN INTERNATIONAL JOURNAL

\title{
An Efficient Human Identification through MultiModal Biometric System
}

\author{
K. Meena ${ }^{1} *$, N.Malarvizhi ${ }^{1}$. \\ ${ }^{I}$ Department of Computer Science and Engineering, Veltech DR.RR \& DR.SR Technical University, Chennai, India.
}

\begin{abstract}
Human identification is essential for proper functioning of society. Human identification through multimodal biometrics is becoming an emerging trend, and one of the reasons is to improve recognition accuracy. Unimodal biometric systems are affected by various problemssuch as noisy sensor data,non-universality, lack of individuality, lack of invariant representation and susceptibility to circumvention.A unimodal system has limited accuracy. Hence, Multimodal biometric systems by combining more than one biometric feature in different levels are proposed in order to enhance the performance of the system. A supervisor module combines the different opinions or decisions delivered by each subsystem and then make a final decision. In this paper, a multimodal biometrics authentication is proposed by combining face, iris and finger features. Biometric features are extracted by Local Derivative Ternary Pattern (LDTP) in Contourlet domain and an extensive evaluation of LDTP is done using Support Vector Machine and Nearest Neighborhood Classifier. The experimental evaluations are performed on a public dataset demonstrating the accuracy of the proposed system compared with the existing systems. It is observed that, the combination of face, fingerprint and iris gives better performance in terms of accuracy, False Acceptance Rate, False Rejection Rate with minimum computation time.
\end{abstract}

Key words :Multimodal Biometrics; Face; Finger print; Iris; Contourlet transform;Classification; Support Vector Machine; NN classifier.

\footnotetext{
*Author for correspondence: meen.nandhu@gmail.com
} 


\section{INTRODUCTION}

In modern trends, all systems require reliable personal identification schemes to either confirm or determine the identity of an individual. The goal of such scheme is to ensure that the provided services are accessed only by the legitimate genuine user. Automated recognition technologies based on individual's behavioral and physiological characteristics are widely in any applications related to security. Biometric systems provide access to physical spaces, information, services and other rights or benefits. Hence, these systems use sensed traits to recognize individual's privacy, legal and sociological factors. The two categories of biometric identifiers include physiological and behavioral characteristics.

A physiological biometric would be identified by one's Face, Iris, voice, DNA or handprint. Behavioral biometrics relates the behavior of a person, such as typing rhythm, gait, voice, hand writing, etc. Biometric technology is now being used in almost every area. A few popular applications of biometrics technology in Government agencies are birth certificates, naturalization papers, passports, Aadhar cards and other government issued documents proven citizenship.

\section{LITERATURE SURVEY}

Snelicket al [1] proposed a multimodal biometric system by combining finger print and Face. Besbes et al [2] proposed a multimodal biometric system using Fingerprint and Iris features. They used a hybrid approach based on Fingerprint minutiae extraction and Iris template encoding through a mathematical representation. Rattani et al [3] proposed a multimodal biometric system using Finger print and Face. They used Scale Invariant Features Transform (SIFT), Fingerprint Verification based on Minutiae matching Technique and Feature Level Fusion for the recognition. They also presented multimodal biometric system based on the integration of face and fingerprint traitsat feature extraction level. Both fingerprint and face images were processed with compatible feature extraction algorithms to obtain comparable features from the raw data. Islam [4] proposed a multimodal biometric system using Ear and Face. They employed Iterative Closest Point (ICP) algorithm, Local 3D feature and Principal Component Analysis (PCA). This approach was very fast and robust against scale variations. CUI et al [5] proposed a multimodal biometric system using Fingerprint and Finger Vein. They applied MHD(Modified Hausdorff Distance)algorithm and Minutiae extraction and matching based on ternary vector. They used score level fusion technique. Muhammad et.al (2011) proposed a multimodal biometric system using Face and Finger Veins. They used LDA methodology for dimensionality reduction. Kazi[6] proposed a multimodal biometric system using Face and Signature. In this system, they used score level fusion techniques to enhance the accuracy rate about 10\% when compared with single Face/signature based biometric system. Radha and Kavitha[7] proposed a multimodal biometric system using Fingerprint and Iris. They employed PCA (Principal Component Analysis) and FLD (Fisher Linear Discriminant) dimensionality reduction techniques for Biometric recognition. Anzar and Sathidevi [8] proposed a multimodal biometric system using Fingerprint and Voice. They used Leave-OneOut Cross Validation technique (LOOCV) and Gaussian mixture model for score level fusion. The performance of the approach is calculated under various noise conditions.

\section{MOTIVATION AND CONTRIBUTION}

From the literature, it is found that, many of the researchers utilized only two biometric features for their experimental investigation. Nowadays, some of the emerging applications like Aadhar card system, uses face, finger and iris features for human authentication. In future, most of the systems combine more than two biometric features for human identity in order to enhance the accuracy. Motivated by this, a new texture based system is proposed by combining very 
familiar biometrics such as face, finger and iris, as they are rich in texture pattern. In order to reduce the time complexity, multiresolution based preprocessing is applied in LDTP and proved as a successful descriptor for face recognition [9]. Multiresolution techniques extract both multi-scale and multi-directivity geometrical structures of the images. In order to capture the intrinsic features such as lines and curves from face, finger and iris images effectively, the proved Contourlet transform technique [10] is used in this paper.

The success of recognition depends on fusion techniques as well as the classification algorithm adopted for investigation. A generic multimodal biometric system may adopt different level of fusion techniques. In general, fusion may be done at sensor level, feature level, match score level, decision score level and rank level. Among that, feature level fusion is mostly used for biometric applications. Motivated and justified by this, LDTP based feature extraction is done in Contourlet domain for face, finger and iris images. Then, feature level fusion technique is applied to generate a feature vector which is compared using NN and SVM classifiers for identification.

\section{ORGANIZATION OF THE PAPER}

The rest of the paper is structured as follows: Section 2 briefly describes the LDTP based feature extraction in detail. Section 3 presents the review about NNC and SVM classifiers. Section 4 focuses on experimental results. Section 5 presents the conclusions and future enhancement of the proposed technique.

\section{PROPOSED SYSTEM}

\section{Outline of the Proposed Approach}

The overall process of the proposed system is illustrated in Figure 1.

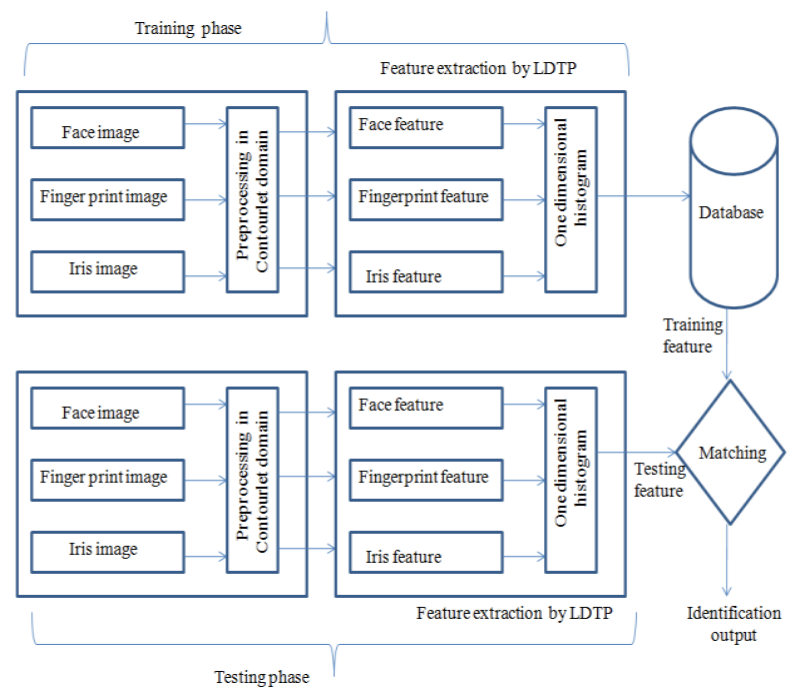

Figure 1.Block diagram of multimodal biometrics recognition system

Multimodal biometrics recognition system consists of two phases namely training phase and testing phase. During training phase, pre-processed image is divided into non-overlapping sub regions of size $N \times N$ and local texture features are extracted by applying LDTP over each region of face, iris and finger print images. For preprocessing, Level 4 Contourlet transform is applied over the images to extract coarse and detail coefficient because it can capture essential features needed for identification. One dimensional histogram constructed for each region are concatenated together to get the global description of an image. In a similar manner, three 1D 
histograms are constructed for face, finger and iris images. Then, all the three histogram are concatenated together and stored as training feature in the database. While in the testing phase, the same procedure is repeated to generate testing feature and is compared against the training feature that is already stored in the database. For comparison, Nearest Neighbor Classifier (NNC) and Support Vector Machine (SVM) classifiers are used.

\section{Feature extraction by LDTP}

The local distributions called textures, characterize object surfaces are used for pattern identification and recognition of images. Face, finger and iris images can be viewed as a texture pattern exhibiting symmetry and regularity. A texture descriptor can characterize an image as a whole as in Gray Level Co-occurrence Matrix (GLCM) or it can also be characterized by local texture descriptor at micro level and through histogram of occurrence frequency of such local descriptors at the macro level.

The micro texture descriptor LDTP is applied over a small local region of size $5 \times 5$ in the image and local texture pattern value which corresponds to that region will be calculated. This descriptor reveals second order local derivative information by encoding the various distinctive spatial relationships contained in the local region. Figure 2(a) shows the local region $I(Z)$ where I represents the gray value of the pixel at position $Z$. Let $Z_{0}$ be a center point and $Z_{i}, i=1,2$, $3 \ldots 8$ be the neighboring pixels around $Z_{0}$. The first order derivative is defined as the absolute difference between a pixel and its immediate neighbor in the right side. The first order derivative $\mathrm{Y}_{0}$ is given as,

$$
I_{o}\left(Y_{o}\right)=\left|I\left(Z_{0}\right)-I\left(Z_{4}\right)\right|
$$

In a similar manner, the first order derivatives are calculated along $45^{\circ}, 90^{\circ}$ and $135^{\circ}$. The same procedure is repeated for all the neighbors to calculate the first order derivative and is shown in Figure 2.The second order derivative of the center pixel is computed between the first order derivative of the center pixel and first order derivatives of all other pixels in its neighborhood. The second order derivative is calculated as,

$$
B_{i}=f\left(I_{\alpha}\left(Y_{0}\right), I_{\alpha}\left(Y_{i}\right)\right), \quad i=1,2, \ldots . .8
$$

\begin{tabular}{|l|l|l|l|l|}
\hline $\mathrm{I}\left(\mathrm{Z}_{20}\right)$ & $\mathrm{I}\left(\mathrm{Z}_{21}\right)$ & $\mathrm{I}\left(\mathrm{Z}_{22}\right)$ & $\mathrm{I}\left(\mathrm{Z}_{23}\right)$ & $\mathrm{I}\left(\mathrm{Z}_{24}\right)$ \\
\hline $\mathrm{I}\left(\mathrm{Z}_{19}\right)$ & $\mathrm{I}\left(\mathrm{Z}_{1}\right)$ & $\mathrm{I}\left(\mathrm{Z}_{2}\right)$ & $\mathrm{I}\left(\mathrm{Z}_{3}\right)$ & $\mathrm{I}\left(\mathrm{Z}_{9}\right)$ \\
\hline $\mathrm{I}\left(\mathrm{Z}_{18}\right)$ & $\mathrm{I}\left(\mathrm{Z}_{8}\right)$ & $\mathrm{I}\left(\mathrm{Z}_{0}\right)$ & $\mathrm{I}\left(\mathrm{Z}_{4}\right)$ & $\mathrm{I}\left(\mathrm{Z}_{10}\right)$ \\
\hline $\mathrm{I}\left(\mathrm{Z}_{17}\right)$ & $\mathrm{I}\left(\mathrm{Z}_{7}\right)$ & $\mathrm{I}\left(\mathrm{Z}_{6}\right)$ & $\mathrm{I}\left(\mathrm{Z}_{5}\right)$ & $\mathrm{I}\left(\mathrm{Z}_{11}\right)$ \\
\hline $\mathrm{I}\left(\mathrm{Z}_{16}\right)$ & $\mathrm{I}\left(\mathrm{Z}_{15}\right)$ & $\mathrm{I}\left(\mathrm{Z}_{14}\right)$ & $\mathrm{I}\left(\mathrm{Z}_{13}\right)$ & $\mathrm{I}\left(\mathrm{Z}_{12}\right)$ \\
\hline
\end{tabular}

\begin{tabular}{|c|c|c|c|c|}
\hline $\mathrm{Y}_{20}$ & $\mathrm{Y}_{21}$ & $\mathrm{Y}_{22}$ & $\mathrm{Y}_{23}$ & $\mathrm{Y}_{24}$ \\
\hline $\mathrm{Y}_{19}$ & $\mathrm{Y}_{1}$ & $\mathrm{Y}_{2}$ & $\mathrm{Y}_{3}$ & $\mathrm{Y}_{9}$ \\
\hline $\mathrm{Y}_{18}$ & $\mathrm{Y}_{8}$ & $\mathrm{Y}_{0}$ & $\mathrm{Y}_{4}$ & $\mathrm{Y}_{10}$ \\
\hline $\mathrm{Y}_{17}$ & $\mathrm{Y}_{7}$ & $\mathrm{Y}_{6}$ & $\mathrm{Y}_{5}$ & $\mathrm{Y}_{11}$ \\
\hline $\mathrm{Y}_{16}$ & $\mathrm{Y}_{15}$ & $\mathrm{Y}_{14}$ & $\mathrm{Y}_{13}$ & $\mathrm{Y}_{12}$ \\
\hline
\end{tabular}

Figure 2 (a)First order derivatives (b) Second order derivatives

The ternary coding function is defined as,

$$
f\left(I_{\alpha}\left(Y_{0}\right), I_{\alpha}\left(Y_{i}\right)\right)=\left\{\begin{array}{l}
0, I_{\alpha}\left(Y_{i}\right) \text { and } I_{\alpha}\left(Y_{0}\right) \leq T \\
1, I_{\alpha}\left(Y_{i}\right) \text { and } I_{\alpha}\left(Y_{0}\right)>T \\
9, \text { elsewhere }
\end{array}\right\}
$$

$\mathrm{T}$ represents adaptive threshold. The LDTP for the pixel $\mathrm{Z}_{0}$ at $\alpha$ directionis computed as follows.

$$
\operatorname{LDTP}\left(I_{\alpha}\left(Y_{0}\right)\right)=\sum_{i=1}^{8} B_{i} * 2^{i}
$$


The Equation (4) shows the value that characterizes the textures of the pixel $\mathrm{Z}_{0}$. The computation of LDTP from a sample region is illustrated in Figure 3.and the detailed explanation about feature extraction is given in [9].

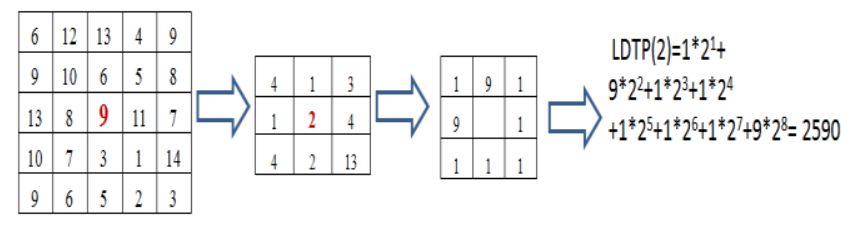

Figure 3.Compuation of LDTP from a sample region

\section{DESCRIPTION OF CLASSIFIERS \\ Nearest Neighbor Classifier}

Nearest Neighbor Classifier (NNC) is used as a one of the predominant classifier in pattern recognition problem. The goal of the NNC is to match a feature of a testing image with that of a training image. A major issue in NNC design is measuring the similarity. There are two possible ways to measure the similarity: one with distance measures and the other with similarity measures. These two measures are the inverse of each other. There exist several similarity and distance measures. In this paper, $\mathrm{NN}$ classifier with G-statistic distance metric is used for classification purpose. The steps for $\mathrm{KNN}$ algorithm is as follows:

* Choose the parameter $\mathrm{K}=$ number of nearest neighbors beforehand.

* Calculate the distance between the testing feature and the training feature using any one of the standard distance measure. Here G statistic is used as distance metric and is given by,

$$
G=2\left[\begin{array}{l}
{\left[\sum_{s, m} \sum_{i=1}^{n} f_{i} \log f_{i}\right]-\left[\sum_{s, m}\left(\sum_{i=1}^{n} f_{i}\right) \log \left(\sum_{i=1}^{n} f_{i}\right)\right]} \\
-\left[\sum_{i=1}^{n}\left(\sum_{s, m} f_{i}\right) \log \left(\sum_{s, m} f_{i}\right)\right]+\left[\left(\sum_{s, m} \sum_{i=1}^{n} f_{i}\right) \log \left(\sum_{s, m} \sum_{i=1}^{n} f_{i}\right)\right]
\end{array}\right.
$$

Where ' $\mathrm{s}$ ' is the histogram of the testing sample and ' $\mathrm{m}$ ' is a histogram of the texture measure distribution of the training image, ' $n$ ' is the total number of bins in a histogram and 'fi' is the frequency at bin ' $i$ '.

* Sort the distances for all the training samples and determine the K number of nearest neighbor based on the minimum distance.

* Use the majority of nearest neighbors as the prediction value.

\section{Support Vector Machine}

The SVM [11] is a binary classifier using the supervised learning paradigm that constructs a decision boundary from the training inputs of two classes. This classifier aims at maximizing the discrimination margin, which is the distance between the decision boundary and the training samples closest to the margin. Training samples used to establish at decision boundary are referred to as support vectors. For linearly separable data, there exists an optimal boundary hyper plane that separates the two classes, class 1 and class 2 .

The complete platform is generalized into a nonlinear case in which the samples are mapped into a suitable high-dimensional space called feature space. Building a separating hyper plane in the said space results the construction of a nonlinear decision boundary in input space. Given that the dimensionality of feature space can be very high, the SVM adopts the kernel function. There are assorted kernel functions - polynomial, linear, sigmoid, and Radial Basis Function (RBF). In Multimodal recognition systems using local texture descriptors, the input to the SVM 
is a global description of the image in the form of a $2 \mathrm{D}$ histogram, and the output is the class label.

\section{EXPERIMENTAL AND PERFORMANCE EVALUATION}

\section{Database Description}

To evaluate the performance of multimodal biometric system, a new database containing 200 set of images are created by combining face images from JAFFE, finger print images from FVC 2000 and iris images from CASIA database. The database consists of 200 individuals with four face, fingerpint and iris images per person. The first face, fingerprint and iris combination is used for training $(200 \times 3=600)$ and the rest three image pairs $(200 \times 3 \times 3=1800)$ are used for testing. In total, 2400 images are used for verification. The experiments were conducted in four sessions recording FAR, FRR and Accuracy and the average is tabulated.

\section{Performance Metrics}

The following performance metrics are used in this paper to measure the effectiveness of the proposed technique. The closest match with any of the correct training image has been considered as the correct match of the test image. The Recognition Rate (RR) is calculated by,

$$
\mathrm{RR}=\frac{\text { Number of correctimages }}{\text { Number of test images }} * 100
$$

In a detection system, two statistics are important: true positives and false positives. True Positive represents the case that was positive and predicted as positive (persons are correctly identified) and False Positive means the case that was negative but predicted as positive (incorrect person identification). An ideal system would have very high true positive and very low false positive rates.

$$
\begin{aligned}
& \text { Precision }=\frac{\text { True Positive }}{(\text { True Positive }+ \text { False Positive })} \\
& \text { Recall }=\frac{\text { True Positive }}{(\text { True Positive }+ \text { True Negative })}
\end{aligned}
$$

\section{EXPERIMENTAL EVALUATION AND ANALYSIS}

\section{Experimental Results by Varying the Number of Training Images}

In this paper, own data set is created for face, finger and iris images from JAFFE, FVC 2000 and CASIA databases. In order to calculate the effectiveness of the proposed technique, number

\begin{tabular}{|c|c|c|c|c|}
\hline & & Recog & ition Rate (\% & \\
\hline & $\mathrm{T}$ & LDTP & features in & Contourlet \\
\hline raining & & Domain & & \\
\hline images & & NN & & SVM \\
\hline & & Classifier & Classi & fier \\
\hline 0 & 5 & 40 & & 43 \\
\hline 00 & 1 & 61 & & 64 \\
\hline 50 & 1 & 91 & & 92 \\
\hline 00 & 2 & 93 & & 95 \\
\hline
\end{tabular}
of training images is varied and the results are represented in Table 1.

Table 1.Recognition Rate of Multimodal Biometrics by Varying Number of Training Images 
Table 1, is found that, the increase in number of training images increases the recognition rate. It is due to the fact that, more textural can be captured when the number of images are increased.

The efficiency of the proposed technique is analyzed in different dimensions by varying the number of testing images and the results are exploited in Table 2.

Table 2.Recognition Rate of Multimodal Biometrics with Different Number of Testing Images

\begin{tabular}{|c|c|c|c|c|c|c|c|}
\hline \multirow[b]{2}{*}{ Classifier } & \multicolumn{2}{|c|}{ Images used } & \multicolumn{5}{|c|}{ Performance metric } \\
\hline & $\begin{array}{l}\text { Total } \\
\text { Images }\end{array}$ & $\begin{array}{l}\text { No.of } \\
\text { Correct } \\
\text { Images }\end{array}$ & $\begin{array}{l}\text { True } \\
\text { Positiv } \\
\text { es } \\
\text { (TP) }\end{array}$ & $\begin{array}{l}\text { True } \\
\text { Negative } \\
\text { s (TN) }\end{array}$ & $\begin{array}{l}\text { False } \\
\text { Positives } \\
(\mathrm{FP})\end{array}$ & $\begin{array}{l}\text { Precision } \\
(\mathrm{TP} /(\mathrm{TP}+\mathrm{FP} \\
))\end{array}$ & $\begin{array}{l}\text { Recall } \\
(\mathrm{TP} /(\mathrm{TP}+\mathrm{TN}) \\
)\end{array}$ \\
\hline \multirow{3}{*}{ NN } & 50 & 40 & 30 & 10 & 10 & 0.75 & 0.75 \\
\hline & 100 & 87 & 79 & 8 & 13 & 0.85 & 0.91 \\
\hline & 150 & 147 & 142 & 5 & 10 & 0.93 & 0.97 \\
\hline \multirow{3}{*}{ SVM } & 50 & 44 & 36 & 8 & 6 & 0.81 & 0.82 \\
\hline & 100 & 94 & 90 & 4 & 10 & 0.9 & 0.96 \\
\hline & 150 & 149 & 145 & 4 & 1 & 0.99 & 0.97 \\
\hline
\end{tabular}

From Table 2, it is examined that the precision and recall values are high for images of 150 in both NN and SVM classifiers. The higher the values of recall and precision enhance the performance of the recognition system. The performance of soft classifier (SVM) is better than hard classifier $(\mathrm{NN})$.

\section{Performance Evaluation of Proposed Technique with Existing Methods}

In order to prove the efficiency of the proposed technique, it is compared against the existing techniques [12] and the results are presented in Table 3.This table consists of biometric features used along with the performance metrics FAR, FRR and Accuracy. 
Table 3.Performance Comparison of Various multimodal biometric approaches

\begin{tabular}{|c|c|c|c|c|c|c|}
\hline \multirow[b]{2}{*}{ Authors } & \multirow[b]{2}{*}{ Biometrics used } & \multirow[b]{2}{*}{ Data sets used } & \multirow[b]{2}{*}{$\begin{array}{l}\text { Feature extraction } \\
\text { technique used }\end{array}$} & \multicolumn{3}{|c|}{ Performance metric } \\
\hline & & & & $\begin{array}{l}\text { FA } \\
\mathrm{R} \\
(\%)\end{array}$ & $\begin{array}{l}\text { FRR } \\
(\%)\end{array}$ & $\begin{array}{l}\text { Accura } \\
\text { cy }(\%)\end{array}$ \\
\hline $\begin{array}{l}\text { Dhanashree et } \\
\text { al }\end{array}$ & $\begin{array}{l}\text { Palmprint, palm } \\
\text { vein }\end{array}$ & $\begin{array}{l}\text { Author created } \\
\text { own database }\end{array}$ & $\begin{array}{l}\text { Region of Interest, } \\
\text { morphological } \\
\text { operations, entropy, } \\
\text { Canberra distance }\end{array}$ & $\begin{array}{l}0.0 \\
3\end{array}$ & 1 & 99 \\
\hline Rattani et al. & $\begin{array}{l}\text { Face, } \\
\text { fingerprint }\end{array}$ & $\begin{array}{l}\text { Bench mark } \\
\text { database } 400\end{array}$ & $\begin{array}{l}\text { Minutiae extraction, } \\
\text { normalization, SIFT } \\
\text { feature point extraction, } \\
\text { K-means } \\
\text { cluestering }\end{array}$ & $\begin{array}{l}1.9 \\
8\end{array}$ & 3.18 & 98 \\
\hline Bhagat et al & Palmvein, face & $\begin{array}{l}\text { Author created } \\
\text { own database }\end{array}$ & $\begin{array}{l}\text { Gray level co- } \\
\text { occurrence matrix, Run } \\
\text { length matrix, Statistical } \\
\text { features, moment } \\
\text { invariants }\end{array}$ & 0.5 & 1 & 98 \\
\hline $\begin{array}{l}\text { Krishneswari } \\
\text { et al }\end{array}$ & $\begin{array}{l}\text { Fingerprint, } \\
\text { palmprint }\end{array}$ & $\begin{array}{l}\text { Hong kong } \\
\text { polytechnic } \\
\text { university } \\
\text { palmprint } \\
\text { database, } \\
\text { FVC2002 DB4B } \\
\text { fingerprint } \\
\text { database } \\
\end{array}$ & $\begin{array}{lr}\begin{array}{l}\text { Discrete } \\
\text { transform, }\end{array} & \begin{array}{r}\text { cosine } \\
\text { Bayesnet }\end{array} \\
\text { classifier, } & \text { Information } \\
\text { gain } & \end{array}$ & $\begin{array}{l}1.0 \\
2\end{array}$ & 0.9 & 98 \\
\hline Gayatri et al & Face, palmprint. & $\begin{array}{l}\text { Bench mark } \\
\text { database }\end{array}$ & $\begin{array}{l}\text { Principal component } \\
\text { analysis, eigen feature, } \\
\text { min-max } \\
\text { Normaliztion, } \\
\text { Euclidean distance }\end{array}$ & 0.5 & 1.2 & 95 \\
\hline Mitul et al & $\begin{array}{l}\text { Palmprint, } \\
\text { fingerprint }\end{array}$ & PolyU database & $\begin{array}{l}\text { Gabor filter, Euclidean } \\
\text { distance }\end{array}$ & 0.2 & 1.1 & 87 \\
\hline Jegadeesan & Fingerprint, iris & $\begin{array}{l}\text { Public available } \\
\text { database } \\
\text { (CASIA) }\end{array}$ & $\begin{array}{l}\text { Histogram equalization, } \\
\text { wienter filtering, } \\
\text { Gaussian low pass filter, } \\
\text { gabor filter, } \\
\text { morphological operation }\end{array}$ & 10 & 5.3 & 91 \\
\hline $\begin{array}{l}\text { Proposed } \\
\text { technique }\end{array}$ & $\begin{array}{l}\text { Face, } \\
\text { fingerprint and } \\
\text { iris }\end{array}$ & $\begin{array}{l}\text { Public available } \\
\text { database } \\
\text { (JAFFE, CASIA) }\end{array}$ & $\begin{array}{l}\text { Contourlet transform, } \\
\text { Local Derivative } \\
\text { Texture Pattern texture } \\
\text { descriptor, Nearest } \\
\text { Neighbor classifier, } \\
\begin{array}{l}\text { Support Vector Machine } \\
\text { classifier }\end{array}\end{array}$ & 0.2 & 1 & 99.5 \\
\hline
\end{tabular}


From Table 3.,it is observed that the combination of face, fingerprint and iris method gives maximum accuracy of $99.5 \%$ when compared with existing methods considered in this paper. One more analysis is performed to compute the computational complexity and the results are tabulated in Table 4.

Table 4.The Average Computation Time

\begin{tabular}{lll}
\hline Feature used & $\begin{array}{l}\text { Computation } \\
(\mathrm{ms})\end{array}$ & time \\
\hline Face and finger print & 190 \\
Palm vein and face & 183 \\
Palm print and finger & 195 \\
print & \\
face, finger print and & 182 \\
iris & 182 \\
\hline
\end{tabular}

From Table 4., it is found that the average computation time of one image is less than $0.7 \mathrm{~ms}$, which is fast enough for real time applications.

\section{CONCLUSION AND FUTURE SCOPE}

In this paper, the performance of three familiar physical biometric is analyzed in multiresolution domain with $\mathrm{NN}$ and SVM classifier. In order to prove the efficiency, the proposed technique is compared against the existing methods. The concatenated feature set of face, fingerprint and iris has more discriminating power than the individual feature. It is concluded that, the performance of this combination is better than performance two modalities in terms of increased FAR, FRR and accuracy. The computation time does not increase for verification compared to others techniques investigated in this paper. In future, Color features can also be included to enhance the performance of the system. This proposed technique can be extended to generate unique identity card that can be utilized for various applications such as health insurance, as a proof of identification when logging into bank accounts from a home computer, as a pre-paid public transport ticket, digital signature, online voting, accessing government databases, etc.

\section{REFERENCES}

Robert Snelick, Umut Uludag, Alan Mink, Michael Indovina and Anil Jain.(2005) Large Scale Evaluation of Multimodal Biometric Authentication Using State-of-the-Art Systems. IEEE Transactions on Pattern Analysis and Machine Intelligence, 27, 3, 450455.

Besbes, F.,Trichili, H., and Solaiman, B.(2008)Multimodal biometric system based on Fingerprint identification and Iris recognitionIn Proc. 3rd Int. IEEE Conf. Inf. Commun. Technol.: From Theory to Applications (ICTTA 2008), 1-5. DOI:10.1109/ ICTTA.2008.4530129.

Rattani, D. R.,Kisku, M.,Bicego, and Tistarelli,M.(2007)Feature level fusion of face and finger Biometric IEEE International conference on Biometrics, Theory, Applied systems, 1-6, 2007.

Islam, S.M.S., Bennamoun, M., Mian, A.S.,Davies,d R.(2009) Score Level Fusion of Ear and Face Local 3DFeatures for Fast and Expression-Invariant Human RecognitionICIAR 2009, LNCS 5627, 387-396, 2009.Springer-Verlag Berlin Heidelberg 2009.

Feifei CUI., Gongping YANG.(2011) Score Level Fusion of Fingerprint and Finger Vein Recognition, Journal of Computational Information Systems 7,16, (2011) 5723-5731 5724.

Kazi M. M., Rode Y.S. ,Dabhade S.B., Al-dawla N.N. H.,Mane A.V., Manza R.R. ,Kake 
K.V.(2012) Multimodal Biometric System Using Face And Signature : A Score Level Fusion Approach, Advances in Computational Research ISSN: 0975-3273 \& E-ISSN: 0975-9085, 4,1, 2012.

Radha,N., Kavitha,A. (2012) Rank Level Fusion Using Fingerprint and Iris Biometric Indian Journal of Computer Science and Engineering (IJCSE) ISSN: 0976-5166, 2, 6, 2012.

Anzar S .M.,Sathidevi P. S. (2012)Optimal Score Level Fusion using Modalities Reliability and Separability Measures, International Journal of computer application, 51, 16, 2012.

Meena,K.,Suruliandi, A., and ReenaRose,R. (2014)Face Recognition Based on Local Derivative Ternary Pattern,IETE Journal of Research, 60, 1, 20-32,2014.

Meena,K.,Suruliandi,A.,andReenaRose,R.(2014)Enhancing the performance of texture based face recognition through multiresolution techniques, International Journal of Biometrics, 6, 4,363-386,2014.

Han,B.,andDavis,L.S.(2012)Density-based multifeature background subtraction with support vector machine,IEEE Trans. Pattern Anal Mach Intell, 34, 5, 1017-1023, 2012.

Sorubasree,S.R.,Radha,N. (2014) A survey on Fusion Techniques for Multimodal Biometric Identification, International Journal of Innovative Research in Computer and Communication Engineering, 2, 12, 7493-7497, 2014.

Received: February 03, 2016; Accepted: July 14, 2016 